\title{
LINKING HIPPARCOS TO EXTRAGALACTIC REFERENCE FRAME (Task of Input Catalogue Subgroup 2130)
}

\author{
A.N. Argue \\ University of Cambridge \\ The Observatories \\ Madingley Road \\ Cambridge CB3 OHA, UK
}

ABSTRACT. A review is given of methods that have been proposed for linking the HIPPARCOS Catalogue of star positions to Extragalactic Reference Frames. The methods to be used by the data reduction consortia are outlined briefly.

\section{INTRODUCTION}

The Instrumental Catalogue of positional coordinates of stars produced by HIPPARCOS will be of very high internal accuracy, about $0 " .002$ for positions and annual proper motions, and the degree of homogeneity will be even higher, with local systematic errors of about one-tenth of these amounts. But the Catalogue will initially be without an equator and equinox, and the task of Subgroup 2130 is to select suitable stars to link to an inertial reference frame established by extragalactic objects.

\section{METHODS}

Extragalactic reference frames have been in use in conjunction with VLBI for some years now (Mueller 1981). They provide for high precision monitoring at the 0".001 level of the earth's motions and the deformations of its crust. Herschel suggested using galaxies as references just over two hundred years ago (in 1785), but classical astrometrists were unable to do this on account of the faintness of galaxies. It was not until photography had become well established that calibrations by galaxies became a practical proposition. Wright at the Lick Observatory carried out tests in 1919, a suitable wide field four element lens, of aperture $51 \mathrm{~cm} \mathrm{f/7}$, was designed by Ross in 1921, and after some delay involving finance etc. the survey got under way in 1947. Meanwhile Russian astronomers had been thinking along similar lines. Deutsch had successfully measured proper motions in SA 80 with reference to NGC 4262. Using the Carte du Ciel type Astrograph with $2^{\mathrm{O}}$ $\times 2^{\mathrm{O}}$ field, he was not able to photograph galaxies either in as large numbers or as faint as could the Lick astronomers with their $6^{\mathrm{O}} \times 6^{\mathrm{D}}$ 
Astrographs. The Russian approach has been to use brighter galaxies selected from lists, and the Lick the more numerous fainter anonymous galaxies showing up on their plates.

The Lick programme has now been nearly completed (Klemola et al 1987). The Northern Proper Motion Catalogue (N. of Dec. - 20 $0^{\circ}$ contains proper motions, positions and magnitudes for over 300,000 stars. There will be very substantial overlap with HIPPARCOS in the range $9^{\mathrm{m}}$ to $11^{\mathrm{m}}$.

Other programmes of absolute proper motions relative to galaxies are being conducted at Kiev, Tautenburg, Shanghai, Bordeaux and other observatories. For most of these programmes the measuring phase had already progressed too far by the time the use of quasars as reference objects had become established.

The Bonn programme (Brosche 1980) is aimed at bright quasars, measuring their apparent ('fictitious') proper motion relative to stars in various catalogues, to include ultimately HIPPARCOS. In this way the residual rotation of each catalogue relative to these quasars will be derived. This programme uses some old astrographic plates.

Other surveys use fainter quasars and so need larger telescopes than astrographs. In these cases the fields are smaller, about $1^{\circ}$ in diameter, or much less in cases where a CCD has replaced the photographic plate. Then the links to the reference frame are established using one or more telescopes of smaller size: the Hamburg programme using an astrograph of field size $6^{\mathrm{O}} \times 6^{\mathrm{O}}$ with objective grating, the La Palma programme using the $18 \mathrm{~cm}$ Carlsberg Automatic Meridian Circle.

\section{THE FAST/NDAC LINK}

Most of the programmes referred to above have been submitted to HIPPARCOS as projects to be carried out by individuals. As time goes on, and the time base for the measured proper motions increases, so also the accuracy of the measured proper motions increases. In the case of radio astrometry, techniques are developing rapidly and the viability of proper motion programmes using radio stars becoming more firmly established. In the years following the HIPPARCOS flight there ought to be published some valuable discussions on the inertial properties of the HIPPARCOS Catalogue and its relation to VLBI frames. But the HIPPARCOS Catalogue itself cannot be kept waiting that long, and its link to VLBI will be carried out in the course of the data reduction by the two reduction consortia, FAST and NDAC. The methods will not depend mainly on those referred to above, but will use two special surveys for which certain programme stars have been given over-riding priority by the Input Catalogue Consortium INCA:

\subsection{Radio stars:}

150 radio stars have been selected. These are optically bright, mostly about $8^{\mathrm{m}}$ or $9^{\mathrm{m}}$, so are well within the range of HIPPARCOS for accurate measurement, and are spread fairly uniformly over the whole sky (Figure 1a). Being members of our own galaxy they share in its motions which must therefore be related to extragalactic systems. This can be done 
very accurately by VLBI and VLA. All have been detected as radio emitters, but not all have yet been confirmed as suitable for good radio astrometry. Nevertheless the indications are that at least one half will be on radio astrometry programmes by the time of the flight. The accuracy obtainable by radio interferometry is at least as high as that expected for HIPPARCOS. In the Northern sky the research groups involved are JPL, the VLA and Barcelona; in the Southern, the Parkes-Tidbinbilla and Tidbinbilla-Harteeboesthoek interferometers. On the optical side, astrometry by the automatic meridian circles at Bordeaux and La Palma, and deep structural investigations by $C C D$ at various observatories, are being carried out.

\subsection{Quasars and Space Telescope:}

This method of linking HIPPARCOS stars to quasars has been described by Hemenway (1985). Super-high priority has been given to 160 stars to link to 90 extragalactic objects. Every star will have been examined by speckle interferometry, those showing structure at the resolution limit of Space Telescope (0".05 at $500 \mathrm{~nm}$ ) being rejected since for these ST cannot do astrometry. Most of the extragalactic targets are brighter than $18^{\mathrm{m}}$, were selected from the IAU list of sources recommended for radio-optical linking (Argue et al 1984), and are being examined for optical structure by deep CCD exposures. The IAU list does not contain enough sources south of Dec. $-40^{\circ}$, but this is being made good by Jauncey and his associates in Australia. The distribution on the sky is shown in Figure $1 \mathrm{~b}$.

\section{REFERENCES}

Argue, A.N. et al, 1985. 'A catalog of selected compact radio sources for the construction of an extragalactic radio/optical reference frame', Astron. Astrophys., 130, 191.

Brosche, P., 1980. 'On the Connection of the Radio and Optical Systems of Positions and Proper Motions', Astron. J., 85, 1674.

Hemenway, P.D., et al, 1985. In 'Hipparcos - Scientific Aspects of the Input Catalogue Preparation' Turon, C. \& Perryman, M.A.C., eds), ESA SP-234, 261.

Klemola, A.R., Jones, B.F. and Hanson, Robert B., 1987. 'Lick Northern Proper Motion Program. 1. Goals, Organization, and Methods', Astron. J. (in press).

Mueller, I.I., 1981. 'Reference Coordinate Systems for Earth Dynamics: A Preview', IAU Coll. No. 56, (Gaposchkin, E.M. \& Kolaczek, B., eds), 1. 

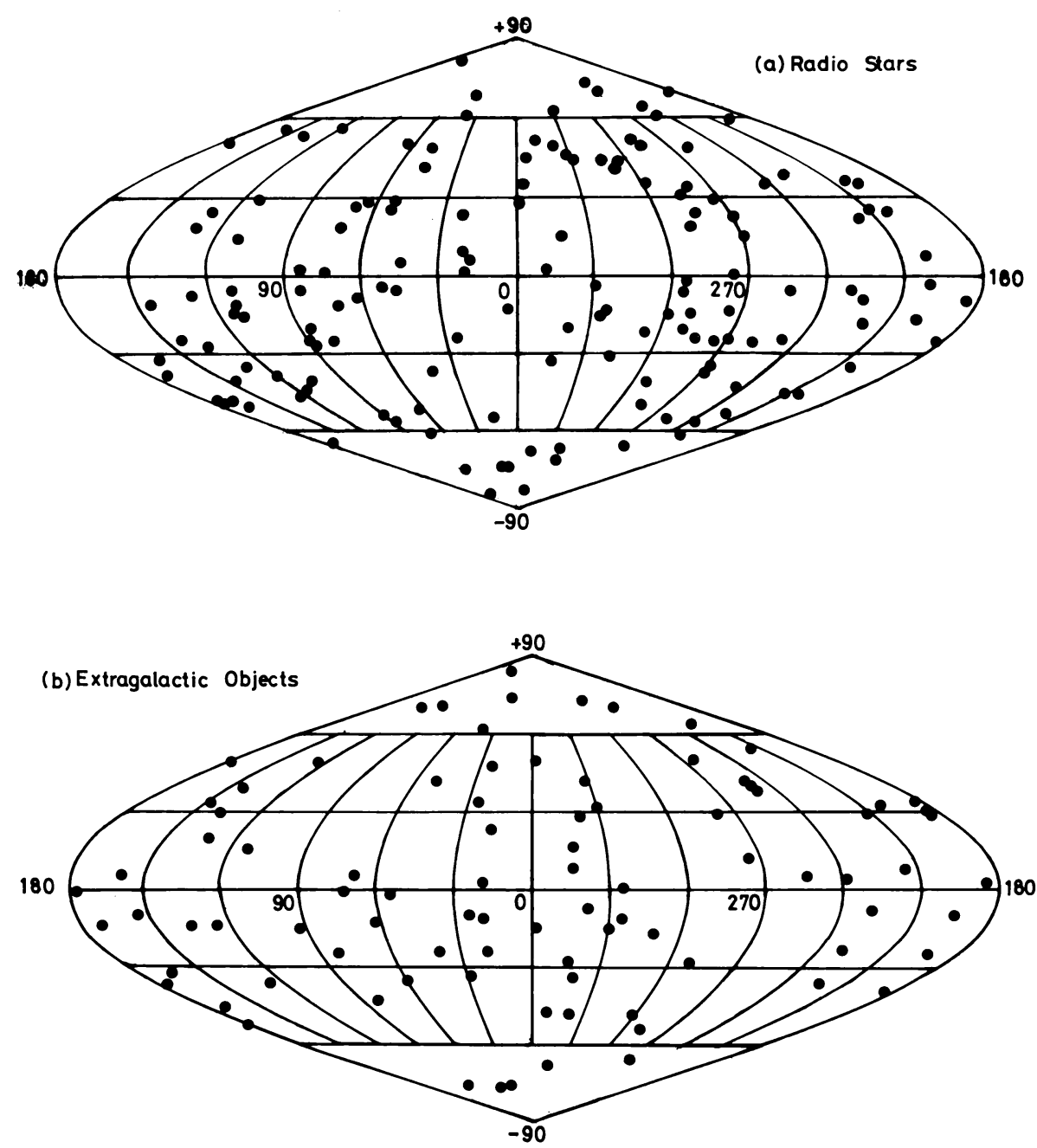

Figure 1. Sky distribution (in Equatorial Coordinates) of Superhigh Priority Objects for linking HIPPARCOS to a VLBI Inertial Reference Frame. 
Discussion:

IASKER

Can you please specify the 1:4

rejection (due to binary stars) more precisely, and on how large a sample was it based?

ARGUE

The important point is that the sample was not a random one - the candidate link stars had first been pre-selected by careful visual examination of Palomar, ESO or UK Schmidt prints and accepted only if apparently "clean". Then on the Anglo-Australian $3.9 \mathrm{~m}$ Telescope, about one in four turned out to be resolved. 